\title{
Dynamics of SEIR Investment Model in Brokerage Business
}

\author{
HyunWook Ryu1)
}

\begin{abstract}
The research model used to predict the spreading path can be interpreted with differential equation(s) and can be applied to various fields. In the field of medical science, studies have covered prediction of the propagation path of diseases (recently including Middle East respiratory syndrome), and estimating extinction probability. Others have utilized the application fields of transportation network in urban engineering, diffusion model of computer virus in such field. Business-wise, the SEIR model can be explained as a feedback effect (recovery) after exposure to a certain event (disease). In this study, we extend the SIR model to financial investment market, specifically in brokerage business, and propose strategic approach. The effectiveness of this model is suggested with theoretical research model and the setting of the variable level for each section. The study demonstrates that the right information at the right time affects the rate of being invested by clients, and re-invested.
\end{abstract}

Keywords : SEIR, Trading Platform, Commission, Brokerage, Investment

\section{Introduction}

Notwithstanding the increased attention to asset management, primary source of revenue comes from commission-based transaction(s) in financial investment business area. In commission-based business, firms profit gets enhanced by getting the clients more frequently, but, at the same time, the firms would have maintain certain level of services to create client loyalty incentives. This is how securities companies in Korea recently provide not only the traditional services as ordering agency but also consulting services to small-to-medium sized enterprises.

While competition is relatively mild, more sophisticated services with strong client loyalty would be the key to success in the market. The firms contemplate ways to develop value-added services to meet their clients' needs, however many have had hard time establishing it. Understanding proper information in timely manner would help identifying effective strategies to step close to the goal. Increasing market share often associated with

Received(April 03, 2019), Review Result(1st: April 23, 2019, 2nd: May 31, 2019), Accepted(June 10, 2019)

1) (Assistant Professor) 11644 Dept. Global Trade and Management, Shinhan University, 95 Hoam-ro, Uijeongbu-si, Gyeonggi, Korea

email: ryuhw@shinhan.ac.kr 
value-added services and high-yield product offerings. As the costs of acquiring new customer mostly exceed the costs of retaining them, injection of relevant information(s) leads to action (or reaction to the proposition made by sales associates). Although customers are not homogeneous as stated in the classical approach of marketing, active and predictive data analytics ensure customer churn and profitability. Finding when and what to put in the trading platform would contribute to significant increases in customer loyalty and the margin growth.

This paper is divided into four parts. The first part presents recent trends in financial asset management business area and literatures previously presented. The second part proposes research model modified to illustrate the flow of investment decision for net-worth individuals. The third section is devoted to the approaches raised to fit in the model developed. The last section concludes.

\section{Business Trends and Literature Review}

\subsection{Asset Management Business Trends in Korea}

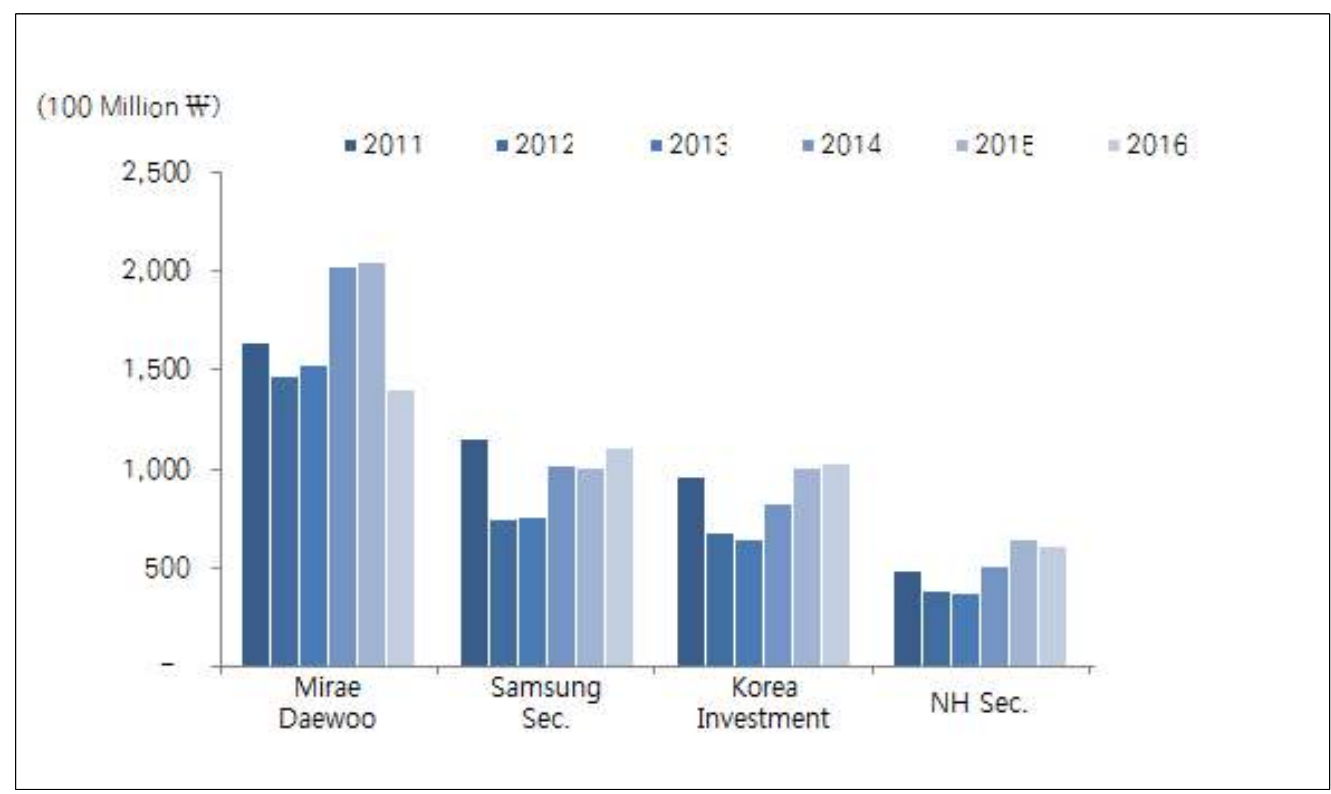

[Fig. 1] Product Sales Margin: the Comparison Year Chart of Major Security Firms (Korea Financial Investment Association, 2017) [1] 
In Korea, financial asset management business are mostly conducted by three entities; (i) commercial banks, (ii) life insurance companies, and (iii) financial investment institutions. The banks primarily handle their depositors as supplementary units, and the insurance companies exercise the business in order to provide their clients an alternative instrument and/or add-on products. Financial investment institutions are better known as securities firms which cover brokerage business, financial advisory services, and investment banking business. As presented with product sales margin in Figure 1, the degree of profit dependence upon fee-based business has been increased over the recent years, meaning that the commission-based business are at the breaking point.

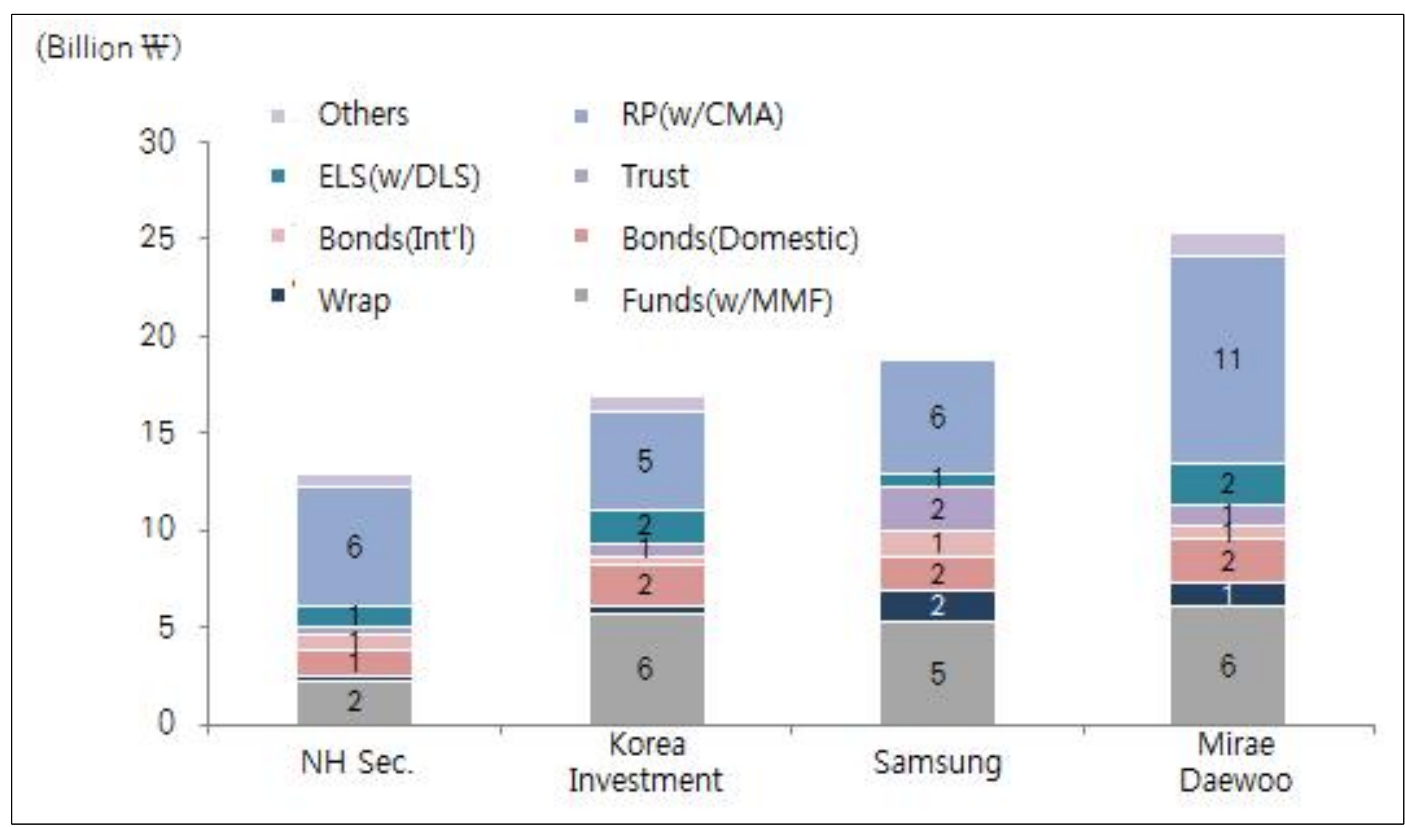

[Fig. 2] Major Security Firms Holding Individual Clients' Assets by Financial Products Offered (Korea Financial Investment Association, 2017) [1]

Many firms have structured organizations from portfolio management perspective, but it does not lead to any change the way to engage with customers. Large-sized space has been introduced but no differentiated service model. Customer rate of return is shown in various customer-access system but evaluation criteria is indifferent. As shown in Figure 2, within the firms' asset compositions, fee-based revenue is less than 30 percent, which is Wrap and Funds (partially). The market understands that it is not about physical inputs such as newly-developed mobile platform or conventional client approaches, but customer-oriented 
innovation strategy. The action plan would have to begin in trading system where one could find the customers' needs, service type, communication tool, value-added solution, advisory services, and evaluation system.

Under the circumstance of the increase in demands for portfolio and tailored asset management services along with powerful digital platform for the self-investors, firms redefine customer-centered innovation for heightening mid-to-long strategies.

Firms figure to make profit structure as the clients achieve their goals by delicate response to the clients' needs, provision of tailored solution, competitive products, and management channel with various and rational costs.

\subsection{Literature Review}

People make decision based on the information, and, in often times, firms would not know how to raise the probability of investment by when and what to provide during the decision-making process. Analyzing information flow has become very important as firms would have to understand the factors on which the clients' decisions are based. The previous studies reviewed are two-fold; one is determinants findings in regard to customer retention or loyalty, and the other one is the model that explains the information and decision(s) made.

The model of SIR (Susceptible-Infectious-Recovered) is primarily developed in biological and medical field in order to study the contagion through the interaction among people. Most studies working on SIR model are inspired by the original work in that a disease is spread from a certain fixed population being susceptible to those infected, and a rate of probability being recovered. Early epidemiology study is conducted to project plague and cholera with three primary compartments in 1927[2]. Later, one study figures the probability of eradicating certain diseases could be varied depending upon the level of risk behavior[3]. More studies have further developed the epidemiology with the basic model investigating dengue transmission, West Nile virus and the event under pulse vaccination incorporated[47]. Other researches utilize modified models such as SIS (Susceptible-Infectious-Susceptible) for determining endemic infrction levels and SEIR (Susceptible-Exposed-Infected-Recovered) for studying sequential process of epidemic in the United States with the data from Google Flu Trends[8-10]. The model is utilized in other fields of study, the information-technology where the SIR model is used to run simulations and evaluate the performances of large-scaled complex computer network[11]. In social network, two researches are done to study advertising spreads and information flows over the network[12-13]. 
A variation model of SIS is proposed in studying the spread of information over social networks, and over Blogspace[14-15].

Since the investment decision is made with information-based approach, this study is similar with such researches with information source detection and/or viral marketing (or SNS) utilizing SIR model[16]. Some studies discuss behavioral approaches and rational decisions by establishing the basic and modified models. And, few paper analyzes consumer behavior in financial sector, and this study is the first to establish and modify the modeling approach to commission-based transaction business[17].

\section{The Model Established}

\subsection{Basic Model}

The basic model is comprised of three compartments; (i) the proportion of individuals who are susceptible to a disease, (ii) the proportion of susceptible individuals who become infectious, and (iii) the proportion of infectious individuals who have recovered.

$$
\begin{aligned}
& \frac{d S}{d t}=-f(\beta) \cdot S \cdot I \\
& \frac{d I}{d t}=f(\beta) \cdot S \cdot I-f(\gamma) \cdot I \\
& \frac{d R}{d t}=f(\gamma) \cdot I
\end{aligned}
$$

Beta is the rate of contact from the given population of being susceptible, and gamma is the rate of recovery. Following studies have conducted on more detailed dynamics with natural death rate and the rate of death caused by diseases.

\subsection{The Decision-making Model Formulated for the Capital Market}

As the SIR model has originally been employed to present contagion effects over a given population, the same mechanism is adopted to explain client investment behavior, in other words, investment decision made. Given a population of size $N$ representing those with excess money, the flow of compartmental classes for investment decision are demonstrated and shown 
in Figure 3: (i) Susceptible to investment (whether or not one has surplus cash), denoted in $S$, (ii) Exposed to a investment system (open an account and join a securities firm but not yet invested), denoted in $E$, (iii) Invested (in other words, a decision's made), denoted in $I$, (iv) Re-invested (or make additional investment), as in $R$, respectively.

Under the assumptions of any given population is all susceptible, and the parameter $\delta$ is greater than zero. The parameter $\alpha$ is the incidence rate of secured individuals to a firm (or a firm's account; over the marketing strategies), and $\beta$ is the rate at which exposed individuals become 'the Invested'; in this stage, the parameter has information valuable enough to draw investors' decision. Finally, the parameter $\gamma$, represents the probability that the invested individuals make additional and/or re-investment.

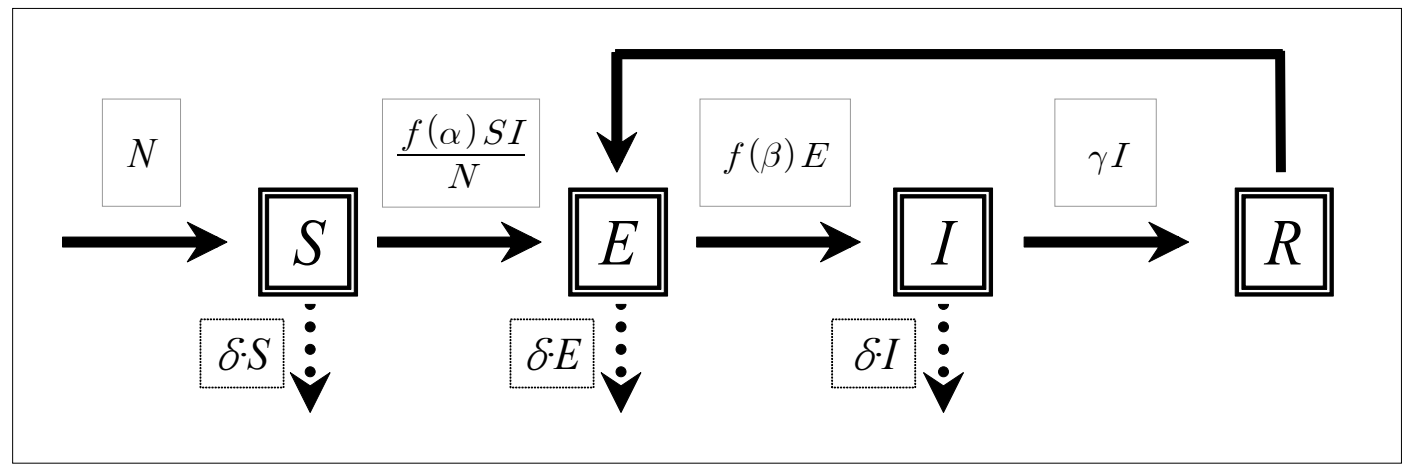

[Fig. 3] Diagram of Transmission Dynamics in Investment Decision Process

In brokerage transaction, more simplified model could be established in order to figure information flow and decision process. As stated in the formula (2), the Exposed-InvestedExposed (EIE) model explains the transmission dynamics for capital investment under commission-based business. The process begins with already-exposed individuals to trading platform where one could access to a range of investments including stocks, bonds, ELS (Equity-Linked Securities), RP (Repurchasing Agreement). The exposed individuals could also receive the market information with performances of many products (via external links or internal algorithm), and order options. Firms put a link on various outside sites

$$
\begin{aligned}
& \frac{d E}{d t}=f(\gamma) \cdot I-f(\beta) \cdot E \\
& \frac{d I}{d t}=f(\beta) \cdot E-f(\gamma) \cdot I
\end{aligned}
$$


where $\beta$ and $\gamma$ denote the force of investment and the rate of satisfaction from the previous investment respectively.

The parameter beta is the transmission rate for the number of contacts per a time in effect, multiplied by proportion of per capita population. The formula (2) states that the probability of transition to the Invested varies with contact rates and relevance of information. The invested individuals keep receive and interpret the information from the system until one transaction completes by placing a sell-order or buy back in RP trades. The investment process flows as one becomes a client belonging to certain security firm. The client is in the state of being susceptible, in other words, being exposed to a trading platform. The degree to which a client makes transaction depends on the quality of information(s). When the client receives relevant information and is satisfied enough, a transaction occurs under the market hypothesis assumed. In the aftermath of a transaction occurrence, the client is back on the state prior to the transaction. Here, the degree to which the client makes additional transaction is up to the investment result and/or the satisfaction level during the prior decision-making process.

\section{Conclusion}

The modelling efforts undertaken so far to study the impact of human behaviour on the spread of infectious diseases were based on varying amounts of anecdotal evidence and common sense, but almost never validated with empirical examinations. Still, these efforts have at least shed some light on the potential impact of behavioral changes on disease dynamics.

An investor's decision to adopt a new behavior often rely on the distribution of similar choices observed within a system network. When determining a financial product (or options), information, differentiated and tailored, may have significant impact on individual behavior of investment decision. In the process of transmission dynamics, information plays pivotal role, which means individuals make investment decision based on how useful the informations are and how those are delivered in effective manner. This study develops the first theoretical model of SEIR for commission-based transaction in financial investment field. In such transactions, individuals rely heavily on various kinds of information so one could determine when, what and how much to place. Once the order is placed, other things including returns being equal, the relevancy and interpretation algorithm of market informations become the key variable to the probability of transition parameters.

SIR model has long been utilized to understand the dynamics of diseases transmission, and 
no academia yet apply to financial area. Although the developed model is limited to brokerage business, it may contribute to the strategy of developing information and contacting the clients. Further study shall complete this model with empirical evidence.

\section{Acknowledgement}

This work was supported by Shinhan University research fund of 2019.

\section{References}

[1] Capital Market Statistics, Korea Financial Investment Association, (2017), Available From: www.kfia.or.kr, (accessed July, 2018)

[2] W. O. Kermack and A. G. McKendrick, Contributions to the Mathematical Theory of Epidemics-I, Proceedings of the Royal Society, (1927), August 1; London, Great Britain

[3] S. M. Blower and A. R. McLean, Prophylactic vaccines, risk behavior change, and the probability of eradicating HIV in San Francisco, Science, (1994), Vol.265, No.5177, pp.1451-1454.

[4] A. d'Onofrio, P. Manfredi and P. Manfredi, Bifurcation Thresholds in an SIR Model with Information-Dependent Vaccination, Mathematical Modelling of Natural Phenomena, (2008), Vol.2, No.1, pp.26-43.

[5] Z. Feng and J. Velasco-Hernandez, Compttitive exclusion in a vector-host model for the dengue fever, Journal of Mathematical Biology, (1997), Vol.35, No.5, pp.523-544.

[6] M. Lewis, J. Renclawowicz and P. van den Driessche, Traveling Waves and Spread Rates for a West Nile Virus Model, Bulletin of Mathematical Biology, (2006), Vol.68, No.1, pp.3-23.

[7] B. Shulgin, L. Stone and Z. Agur, Pulse Vaccination Strategy in the SIR Epidemic Model, Bulletin of Mathematical Biology, (1998), Vol.60, No.6, pp.1123-1148.

[8] S. E. Chick, A. L. Adams and J. S. Koopman, Analysis and simulation of a stochastic, discrete-individual model of STD transmission with partnership concurrency, Mathematical Biosciences, (2000), Vol.166, No.1, pp.45-68.

[9] V. Dukic, H. F. Lopes and N. G. Polson, Tracking Epidemics With Google Flu Trends Data and a State-Space SEIR Model, Journal of the American Statistical Association, (2012), Vol.107, pp.1410-1426.

[10] P. E. Lekone and B. F. Finkenstadt, Statistical Inference in a Stochastic Epidemic SEIR Model with Control Intervention: Ebola as a Case Study, Journal of the International Biometric Society, (2006), Vol.62, No.4, pp.1170-1177.

[11] D. Chen, L. Li, M. Shang, Y. Zhang, T. Zhou, Identifying influential nodes in complex networks, Physica A, (2012), Vol.391, No.4, pp.1777-1787. 
[12] Y. Jing, L. Peiyu, T. Xiaobing and L. Wenfeng, Improved SIR Advertising Spreading Model and Its Effectiveness in Social Network, Procedia Computer Science, (2018), Vol.129, pp.215-218.

[13] F. Wu, B. A. Huberman, L. A. Adamic and J. R. Tyler, Information flow in social groups, Physica A, (2004), Vol.337, No.1, pp.327-335.

[14] A. Guille, H. Hacid, C. Favre and D. A. Zighed, Information diffusion in online social networks: a survey, SIGMOD record, (2013), Vol.42, No.2, pp.17-28.

[15] D. Gruhl, R. Guha, D. Liben-Nowell and A. Tomkins, Information Diffusion Through Blogspace, The $13^{\text {th }}$ International World Wide Web Conference (WWW'04), (2004), May 17-20; New York, USA

[16] K. Sohn, J. T. Gardner and J. L. Weaver, Viral Marketing - More than an Buzzword, Journal of Applied Business and Economics, (2013), Vol.14, No.1, pp.21-42.

[17] H. Ryu, A Study on SIR information model and its application to Financial Institutions, Asia-pacific Journal of Multimedia Services Convergent with Art, Humanities, and Sociology, (2018), Vol.8, No.8, pp.313-320. 